\title{
Bolt-connected external ventricular drainage: The key to a better accuracy and less complications?
}

\author{
Parmenion P. Tsitsopoulos ${ }^{1}$ \\ Received: 13 November 2018 / Accepted: 15 November 2018 / Published online: 21 November 2018 \\ (C) Springer-Verlag GmbH Austria, part of Springer Nature 2018
}

The use of external ventricular drainage (EVD) has been one of the commonest procedures in everyday neurosurgery and a valuable tool for neurosurgeons and neurointensivists [6]. It is more frequently applied in the emergency setting either as a life-saving operation or for monitoring and controlling intracranial pressure (ICP). Although in most occasions, it is used temporarily, the eventual need for permanent diversion and controlled drainage of the cerebrospinal fluid (CSF) can also be required [3].

The traditionally tunneled technique has been initially described around 40 years ago and it is still the most commonly used worldwide [5]. After a skin incision and a burr hole usually in the non-dominant hemisphere, the catheter is inserted with freehand technique to the frontal horn. The catheter is then tunneled under the skin and externalized at a point where it is fixed with sutures. Next, the catheter is connected to a large volume bag and typically, to an ICP-monitoring system [6]. As expected, placement is not complication free as numerous problems can be encountered. Catheter misplacement, CSF leakage, wound and CSF infection, catheter migration and disconnection, intracranial bleeding, and neurological deficits have been repeatedly reported among others [4]. Further, not infrequently, reoperation cannot be avoided which can result in prolonged length of stay, increased costs, and morbidity.

For about a decade, bolt-connected EVDs have been used in clinical practice in Denmark with success $[2,6]$. The technique is quite different to the conventional tunneled. The catheter is initially cut to a length of $55-65 \mathrm{~mm}$, is then attached to the distal metal tip of the bolt, and secured with a non-

This article is part of the Topical Collection on CSF Circulation

Parmenion P. Tsitsopoulos

ptsitsopoulos@auth.gr

1 Department of Neurosurgery, Hippokratio General Hospital, Medical Faculty, Aristotle University School of Health Sciences,

Thessaloniki, Greece absorbable suture. A stylet is inserted in the system which is followed by puncture of the ventricular system. The bolt is screwed into the burr hole and secured until it reaches an optimal fit. Lastly, the proximal end is shaped for a secure drain-fit with a Luer-Lock [2].

Bergdal et al. in 2013 tested the bolt-connected EVD in 97 patients. This study sample was compared with 57 patients who received a tunneled EVD. In the bolt-connected group, placement was statistically more accurate compared to the tunneled group. Patients subjected to the bolt-connected EVD had less reoperations compared to the tunneled group (2.1\% vs. $14 \%$ ) [2]. In 2016, Jensen et al. compared the two techniques in a larger number of patients, 141 and 131, for the bolt-connected and the tunneled group, respectively. It was found that bolt-connected EVDs had significantly less CSF leakage and significantly less complications requiring reoperation for EVD (e.g., mechanical problems) compared to tunneled EVDs. However, no difference in the infection rate was found between the two groups [6].

Assad and Bjarkam recently compared the two aforementioned techniques in a smaller patient sample $(n=49)$. This time, the study material was collected prospectively. Again, significantly more complications were seen in the tunneled group (including also infections) where it was found a 3.4 times higher risk for complications compared to the boltconnected group. None patient required revision in the boltconnected group, whereas, $17.4 \%$ of cases, the EVD was revised in the tunneled group. According to the authors, bolttunneled EVD should be the first choice, with the traditional tunneled method retaining its role in pediatric patients as well as in occipital insertion and through craniotomies [1].

Although the results are again in favor of the boltconnected EVD, the latter study has problems that raise skepticism and its findings should be interpreted with caution. The level of surgeon's experience is unclear. Different surgeons with other levels of experience were likely interfered. Few patients received the bolt-connected $\operatorname{EVD}(n=12)$ which cannot allow clear conclusions, similarly, when someone is trying 
to compare the two drain types. The fact that placement accuracy could not be evaluated in a notable number of patients is another drawback since there are larger studies published in the literature comparing these two types of EVD. Likely, recruitment of more patients from both groups (especially for the bolt-connected EVD) in a more organized way and for at least one more year will likely result in more robust findings and conclusions.

Bolt-kit EVDs have been used by other groups with similar success [7]. It is a less-traumatic technique easily performed even by inexperienced neurosurgeons which, assuming that EVD care is similar in all occasions, it can reduce morbidity and financial costs. Since less problems seem to occur, there is some evidence to support the superiority of bolt-connected drains over the traditional tunneled drains. Moreover, the continuously growing use of neuronavigation during EVD insertion will increase placement accuracy and further strengthen its value. However, it should be stressed that, currently, most data are derived from retrospective and not strictly controlled material which is subject to known weaknesses. Therefore, more evidence is needed from prospective studies in different regions and practices before the use of bolt-connected devices becomes common practice in neurosurgery.

\section{References}

1. Asaad SK, Bjarkam CR (2018) The Aalborg Bolt-Connected Drain $(\mathrm{ABCD})$ study: a prospective comparison of tunnelled and boltconnected external ventricular drains. Acta Neurochir (in press)

2. Bergdal O, Springborg JB, Holst AV, Hauerberg J, Way S, Breum P, Romner B (2013) Accuracy of tunnelated vs. bolt-connected external ventricular drains. Clin Neurol Neurosurg 115:1972-1975

3. Cinibulak Z, Aschoff A, Apedjinou A, Kaminsky J, Trost HA, Krauss JK (2016) Current practice of external ventricular drainage: a survey among neurosurgical departments in Germany. Acta Neurochir 158:847-853

4. Fried HI, Nathan BR, Rowe AS, Zabramski JM, Andaluz N, Bhimraj A, Guanci MM, Seder DB, Singh JM (2016) The insertion and management of external ventricular drains: an evidence-based consensus statement : a statement for healthcare professionals from the Neurocritical Care Society. Neurocrit Care 24:61-81

5. Friedman WA, Vries JK (1980) Percutaneous tunnel ventriculostomy. Summary of 100 procedures. J Neurosurg 53: $662-665$

6. Jensen TS, Carlsen JG, Sorensen JC, Poulsen FR (2016) Fewer complications with bolt-connected than tunneled external ventricular drainage. Acta Neurochir 158:1491-1494

7. Schodel P, Proescholdt M, Ullrich OW, Brawanski A, Schebesch KM (2012) An outcome analysis of two different procedures of burr-hole trephine and external ventricular drainage in acute hydrocephalus. J Clin Neurosci 19:267-270 